\title{
CATACLYSMIC VARIABLES AS BINARY STARS: THEN AND NOW
}

\author{
ROBERT P. KRAFT \\ University of California Observatories/Lick Observatory \\ Board of Studies in Astronomy and Astrophysics \\ University of California, Santa Cruz, CA 95064
}

\begin{abstract}
A brief history is given of the clues that led to the establishment, in the period c. 1950 to 1965 , of a universal binary model for novae and related cataclysmic variables. The observational facts, established early in this period by A. H. Joy and R. F. Sanford (selected binary orbits), J. L. Greenstein (spectroscopy) and $\mathrm{M}$. Walker (photometry) are reviewed, as are the theoretical ideas that formed the basis for the early models, viz., those of G. Kuiper (restricted 3-body problem), A. Sandage and M. Schwarzschild (stellar evolution), J. Crawford ("dog-eat-dog" hypothesis), F. Hoyle and H. Bondi (accretion), S.-S. Huang (angular momentum losses), and S. Chandrasekhar (gravitational radiation). Some comparisons are made between "what we knew then and what we know now." With apologies to all, the speaker will recall some anecdotes of an earlier time and comment on developments of the past score of years from a very personal perspective.
\end{abstract}

Just after the Baltimore IAU, I spent a month at STScI as a scientific visitor - a delightful period. I had been freed from administrative chores at Lick and enriched with the opportunity to do some long-deferred work on the stellar content of the Galactic halo. One afternoon, Mike Shara and Mario Livio popped into my office and surprised me with a seemingly odd proposal: would I give the opening talk at a forthcoming IAU Symposium on Novae? I said "You have to be kidding! I haven't worked in the subject for 20 years." The long-and-short of it is that they were serious, I succumbed, and so here I am.

The question is "what's my role here?" Mike and Mario didn't offer any clues and I feel a little like one of those just unwrapped and "sprung-to-life" mummies in a Hollywood B-grade horror movie.

So what I propose to do is to reconstruct for modern astronomers what it was like to be an observer in the period from about 1950 to 1965 , to discuss the observational clues and theoretical ideas that enabled us to stumble on the binary/mass-transfer/accretion-disk model for old novae and related objects, to highlight a little the unanswered questions we thought were important then, and to comment on the developments of the past score of years - at least the milestones that seem important to this old guard worker in the field. This is presumptuous, of course, but I offer it anyway on the grounds that someone who has been out and has now returned to the fold may see things in a special — well, at least "peculiar," light. 
So for a moment, "return with me to those thrilling days of yesteryear," to an era without CCDs, flat-fielding, blocked columns, charge-transfer problems, IRAF, computers that won't boot, point-spread functions, data sets, Komolgorov-Smirnov tests, and Monte Carlo simulations - to a pristine era of photographic plates and DC photometers, long cold nights on dangerous platforms at prime and Newtonian foci, and personal confrontations with God in the dark. A time before those terrible nanometers, when all that was right and holy in the world lay between 3500 and $7000 \AA$. Radio astronomy was in its infancy and the UV was a region in which you looked at the sun for a few moments with a detector mounted on a V2 rocket.

Specifically, let me reconstruct the perspective from Berkeley, the way we saw it in $1952 / 53$. As excuse, I offer the fact that Otto Struve, who had been the chief honcho of binary star astronomy and who had stimulated a whole generation of variable and binary star spectroscopists, had just relocated his school from Yerkes to Berkeley, and in the process had carried a significant fraction of the Yerkes young people with him (e.g., Henyey and Phillips). Meantime, Jesse Greenstein, who had also been at Yerkes, was in the process of gathering up several of the others (Osterbrock, Code, Münch) to staff his new astrophysics group at Caltech. All this succeeded in bringing the latest in theoretical astrophysics to the California institutions where there existed already an established tradition of strength in optical observational techniques - to Mount Wilson, to Lick where the 120-inch was under construction, and to Palomar, at a time when the 200-inch had been in operation for less than a decade. Recall, too, that the world boasted then only a few large telescopes - the 200-inch, the 120-inch (under construction), the 100-inch at Mount Wilson and the 82-inch at McDonald - that's all there were, all of them were in the United States, and most of them in California.

Returning to Berkeley, I should say that I went there as a graduate student in 1951, and in the first years worked for Otto Struve as a research assistant. Those of us who worked for him revered him and a good word from him was the equivalent of a papal benediction. As George Herbig once noted, he made you feel that the rigors of observation were a privilege, not a duty, and that the sacrifices required of you to do the observing were no more than he himself would be willing to endure to do the same. A more-or-less permanent disciple on the scene was the theoretician Su-Shu Huang who, as we will see later, played a decisive early role in the angular momentum problem.

A lot of theoretical notions now taken for granted were either new or unknown in the early $50 \mathrm{~s}$. Nobody had conceived of QSOs or pulsars. The only collapsed states of matter known were white dwarfs; neutron stars hadn't penetrated the surface of general astronomical thought, much less black holes. Photoelectrically-based color-magnitude diagrams of open and a few globular clusters were beginning to appear as 1P21 photomultiplier tubes came into general use. Stellar evolution, i.e., the fundamental understanding of the structure of giants, had just burst on the scene with the appearance of the 1952 Sandage-Schwarzschild [1] paper. Enter fellow Berkeley graduate student John Crawford. He had worked on the Manhattan Project and unlike me, he was a real physicist, having taken a master's degree from University of Chicago in physics. A student of Henyey's, Jack tackled the mysterious problem of the subgiant components of eclipsing binary systems, objects in which the brighter component was usually less massive and later in spectral type than its companion. He showed in a paper written in 1954 [2] that the primaries, statistically at least, appeared to fill their lobe of the inner Lagrangian surface. He hypothesized that they had lost mass through the inner Lagrangian point $L_{1}$ owing to evolutionary swelling following the Sandage/Schwarzschild scenario. The lost mass was supposedly collected by the secondary or lost to the system; anyway, this work constitutes the first appearance of mass-exchange, which Fred Hoyle later dubbed the "dog-eat-dog" hypothesis. 
Meantime, the indefatigable Mount Wilson observer, Alfred H. Joy, who had done so much fundamental pioneering work on faint variable stars discovered, using the 60-inch and 100-inch reflectors at Mount Wilson, that AE Aqr was a double-lined spectroscopic binary, with a period of about $16^{\mathrm{h}}$ [3]. The unusual spectrum (bright lines of $\mathrm{H}, \mathrm{He}$ I and $\mathrm{Ca}$ II together with the absorption lines of a K-type dwarf) was interpreted as belonging to a binary object in which the subluminous component was surrounded by a shell of hot gas expanding at a velocity of $\sim 700 \mathrm{~km} / \mathrm{sec}$. The spectrum proved remarkable similar to that of SS Cyg, which Joy demonstrated a little later [4] to be a spectroscopic binary with the remarkably short period of $6^{\mathrm{h}} 38^{\mathrm{m}}$.

Add to this Roscoe Sanford's discovery in 1948 [5] that the nova T CrB was also a member of a binary system containing an M-giant and a hot emission line object, but with the rather long period of $230^{d}$, one began to suspect a generic relationship between the cataclysmic variable phenomenon and the business of being a binary. But what underlying physical process could connect these two phenomena?

Jack Crawford's mass-transfer hypothesis, if it could be extended a little, might just provide the answer, as Jack and I speculated, following Joy's announcement of the binary character of AE Aqr. Suppose that, instead of a picture in which the hot star is surrounded by an expanding low density cloud, you had instead a hot star surrounded by a collapsing cloud. Spectroscopically, you wouldn't be able to tell the difference. Furthermore, we all knew that white dwarfs mostly didn't have hydrogen, except maybe in a thin envelope where nuclear processing could no longer take place. What would happen if the hydrogen-rich red dwarf in such a binary system could spill its fresh hydrogen-rich atmosphere onto a white dwarf? Isn't it possible that an explosion would ultimately take place when the accreted material became hot enough that either the CNO cycle or P-P chain would be set off ?

What justification could one find for taking such a view? Well, astronomy in those days proceeded mostly by metaphor and analogy. Maybe what worked in one situation could be applied to work in another. Thus, it seemed perfectly natural to Joy and other Mount Wilson astronomers, that the hot blue star should be surrounded by an expanding envelope: had not one seen such things in $\mathrm{Be}$ stars and shell stars? Moreover, one expected radiation pressure to drive expanding envelopes, as Eddington and others had predicted.

On the other hand, students have a natural propensity for upsetting quasi-established (or even well-established) ideas, and we were no exception. In the summer of 1953, Herman Bondi came to the Berkeley Astronomy Department as a visiting professor, carrying with him the (then) new gospel of "accretion." Of course, this was the "columnar"-style accretion associated with a star plowing through the IS medium - as he and Fred Hoyle had worked it out, but still the seed idea was there and found fertile ground in the minds of a couple of graduate students. At the same time, the ghost of the Yerkes Observatory tradition via Otto Struve wafted through the halls, as Jack and I studied Kuiper's famous paper on $\beta$ Lyrae, gas streams, Lagrangian surfaces, and the restricted three-body problem. Obviously the dwarf K-type star must fill its inner Lagrangian surface (as in the subgiant components of eclipsing binary systems), spill matter into the other lobe, which then took up residence in a ring around the white dwarf. The ring would have to spread out owing to turbulent diffusion (the term "turbulence" was all the rage then - again the influence of Otto Struve), and the inner part of the ring ultimately would have to fall on the white dwarf. But wait a minute: how can you have a system in which the components have $M_{v} \sim+5$ or +6 , yet one of them is a white dwarf? Easy: we don't see white dwarf lines in the spectrum and the emergent flux comes not from the white dwarf itself but from the conversion into radiation of the kinetic energy of the infalling material, i.e., 


$$
L \propto\left(\frac{G \mathcal{M}}{R}\right) \cdot \dot{\mathcal{M}}
$$

The $\mathcal{M}$ required had to be about $10^{-8} \mathcal{M}_{\odot} /$ yr. Could the $\mathrm{K}$-dwarf provide this mass flux? Yes, provided it was in a state of evolutionary expansion. Jack Crawford worked out, from the Sandage-Schwarzschild models, the rate of increase of radius of the red star, and determined a mass transfer rate again of order $10^{-8} \mathcal{M}_{\odot} / y r$. Finally, it was possible to show that, given an accretion velocity of $\sim 400 \mathrm{~km} / \mathrm{sec}$ (from the observed emission-line widths) and a density worked out from the strength of the hydrogen lines assumed to arise from recombination radiation, one obtained again an accretion rate of order $10^{-8} \mathcal{M}_{\odot} / \mathrm{yr}$. The main thing missing in the picture was a coherent model of the outbursts. We tried to make hydrogen-burning work in a crude calculation, but consistently failed in our naivete (for reasons that became obvious from the solid work of Starrfield, Truran, and others in later years). Our imagination had been fired by Leon Mestel's 1952 [6] paper on accretion of interstellar hydrogen by a white dwarf. The trouble was that you heated the whole white dwarf core by conduction and you got a supernova - an embarrassment of riches, to say the least.

Meantime, a real breakthrough in establishing the binary nature of novae was about to emerge thanks to observations of a quite different sort, i.e., photometric techniques. Merle Walker, who had been a Berkeley Ph.D. early in Struve's tenure there, had undertaken a thesis on the nature of $\beta$ CMa stars, and had developed an active interest in the photometry of rapid variables. (By "rapid" in those days, we meant objects that varied on timescales of order 1 minute to 1 hour.) Walker left Berkeley and Lick around 1953 to take up residence on Santa Barbara Street as a Carnegie Fellow of the Mount Wilson Observatory. Walker's interest in rapid variations was sparked by his work [7] on McRae +43 : 1 (now known as MV Lyr) and by the "flickering" that had been observed in UX UMa, an object that had been found to be a short period eclipsing binary $\left(P=4^{\mathrm{h}} 43^{\mathrm{m}}\right)$ by Linnell as early as 1949 , and which Walker and Herbig [8] had studied quite extensively in the period 1951-53. The spectroscopic and photometric similarity of UX UMa and McRae $43: 1$ to AE Aqr on the one hand and, on the other hand, to a number of old novae that Jesse Greenstein had been studying spectroscopically with the 200-inch, led Walker to initiate a program at Mount Wilson of searching for photometric "flickering" in a number of old novae and cataclysmic variables. This quite immediately paid off, as is demonstrated especially in the case of $\mathrm{T} \mathrm{CrB}$ in IAU Symposium No. 3. But a much bigger pay-off was in the offing. One night in 1954, while observing the famous nova DQ Her (1934) for such flickering, Walker noted a decline in the Brown recorder output which didn't recover, and indeed didn't recover until it had exhibited the extraordinary dip one normally associates with an eclipse. Indeed DQ Her turned out to be an eclipsing binary with a period of $4^{\mathrm{h}} 39^{\mathrm{m}}$ [9]. This result became something of the "toast" of the 1955 Dublin IAU meeting. You have to remember that in the postwar period, Nova Her (1934) was ingrained in every astronomer's mind as the nova - it had been by far the most extensively studied nova in the years following the establishment of the first "giant" telescopes and it was regarded as the classical object of its type.

Of additional interest was Walker's discovery, quite unprecedented in its time, of the 71 second oscillation - with its 0.02 mag amplitude. Quite a feat in its day - nobody had ever heard then of a power spectrum analysis! [It's worth noting here that the interpretation of the phenomenon in those days was that it represented the radial pulsations of the underlying white dwarf. Using pulsation theory and white dwarf theory, one found $\mathcal{M}_{\mathcal{W D}} \sim 0.25 \mathcal{M}_{\odot}$, as opposed to $\mathcal{M}_{\mathcal{W D}} \sim 0.007 \mathcal{M}_{\odot}$ (!) from conventional analysis of the eclipse data. Since nobody believed this, the later rejection of the pulsational hypothesis (for good reasons, of course) was a bit of a blow!] 
After the period at Mount Wilson, Walker moved to Case Institute and then returned to Lick Observatory as a staff member in the late 50 s, where he continued work on the brightness fluctuations of novae and related objects such as RW Tri, and where he discovered the short-period binary characteristic of Nova T Aur (1891) $\left(\mathrm{P}=4^{\mathrm{h}} 54^{\mathrm{m}}\right)$ in 1962 [10]. Meantime, I left Berkeley to become a National Science Foundation Fellow at Mount Wilson. In those days, fellows were not allowed to use the Palomar 200-inch; my attempts to establish orbits for cataclysmic variables of the dwarf nova type - by now it was a reasonable hypothesis that all such objects were binaries - were frustrated by bad weather, faintness of these objects at minimum, and the smallness (!) of the telescopes at Mount Wilson. With the 100 -inch nebular spectrograph (resolution 7 to $10 \AA$ ), you could reach 14th magnitude in about half an hour and have a pretty good spectrogram. (That is about what you can do nowadays with a CCD and suitable spectrograph on a 12-inch telescope!) But I had one lucky stroke - a good series of spectrograms taken in May 1956 provided the first orbit associated with the He II line in Nova DQ Her [11]. This encouraged Jesse Greenstein to examine the object with the nebular spectrograph at Palomar, and in 1959, after I had gone to Indiana and later Yerkes, we published a paper [12] together on the amazing spectrum of DQ Her through the eclipse cycle velocity curve, rotational disturbance, and all. The main results were essentially that if an M-type star was the secondary and filled its Roche lobe, then the AE Aqr model could be carried over to DQ Her. The eclipse was that of a rotating accretion disk by the dark, presumably M-type star, and the mass of the white dwarf that was permitted was certainly in excess of $\sim 0.25 \mathcal{M}_{\odot}$. Left unexplained, however, was how an M-type dwarf could drive mass-loss through $L_{1}$. Surely stellar evolutionary swelling-up could be ruled out. But more about this later.

As a former fellow, I was allowed to return in 1957 to Mount Wilson as a "guest investigator" while still on the Indiana faculty and had an opportunity to re-investigate $\mathrm{T} \mathrm{CrB}$. This object you could reach in long exposures with the 100-inch coude spectrograph. I attempted [13] to reconstruct the hydrogen emission lines of the "blue component" to see if the orbit of a double-lined spectroscopic binary could be recovered. The emission lines were painfully reconstructed by subtracting out the spectrum of a standard M3 III star - all this by photographic techniques on gear-driven paper tapes, in which one had to allow for the non-linearity of the detector. How much more easily this could be done nowadays with linear detectors and digital techniques! It's important, too. Recall that T CrB is the only known case in which the "blue star" mass exceeds the Chandrasekhar limit. Since the M-star velocity amplitude and the period are well known, you must sharply reduce the amplitude of the blue star's radial velocity curve to force the blue star mass to a value below the limit, but just the same a more reliable determination of mass should be undertaken with modern techniques.

I returned to Santa Barbara Street as a staff member in 1960 , now with full access to the 200 -inch. Of course, I immediately embarked on a spectroscopic survey looking for orbits for dwarf and classical novae - and this was a success, I believe [14]. You have an advantage, naturally, using spectroscopy versus photometry, since you don't need to depend on the orientation of the orbit in space to give you eclipses. On the photometric side, I was lucky to have Voytek Krzeminski and George Mumford as collaborators. Of course, it turned out that many old novae and dwarf novae showed velocity variations and yielded orbits - U Gem, Z Cam, RX and RU Peg among the former and along with DQ Her and T CrB, I found orbits also for GK Per, V603 Aql, and WZ Sge, among the latter. Add to this Walker's work on T Aur, plus the earlier studies of nova-like variables such as RW Tri, UX UMa, Herbig's still earlier work on VV Pup, and Joy's work on AE Aqr and SS Cyg - it now seemed pretty certain that all dwarf and classical novae were close binaries in which a white dwarf was accompanied by a late-type star which overflowed its inner Lagrangian surface and which spilled matter into an accretion disk around the white dwarf. 
The most fun I ever had as an astronomer was the discovery of the binary nature of WZ Sge, which at the time of discovery had the shortest known orbital period ( 82 minutes). The charm of going against the grain of the traditional spectroscopists and using a "telescope rate offset-single trailing" technique - which led to the discovery of the so-called "S-wave disturbance" - is too long to go into here, but I do want to mention some of the consequences. The 82 minute period and corresponding small dimensions of the system were striking. Vaguely I remembered attending what seemed at the time like a very esoteric lecture by Chandrasekhar while I was still on the Yerkes staff, just before returning to Pasadena. Chandra had talked about something mysterious, viz., gravitational radiation, which could be emitted on an interesting cosmic time-scale by two white dwarfs if they could be found in a binary system with a sufficiently short period. Could this be applied to WZ Sge? I had no idea where to look for the equations, so I turned to Jesse Greenstein. As those of you know who have ever talked science with Jesse, he is an inexhaustable store of fundamental knowledge about everything and can work out wonderful things on a paper napkin over lunch or dinner. Jesse knew about this, said it was in Landau and Lifshitz and that I ought to talk to Jon Mathews of the Caltech physics department. Thus was born in 1962 [15] what I think is the first paper on gravitational radiation in the ordinary astronomical literature, when the three of us suggested [14] that WZ Sge might radiate gravitational waves on an interesting cosmic timescale. In 1966, I took this up again in a paper [15] in which I argued that gravitational-wave "grinding" might be a means of solving the mass-loss problem, since most of the late-type components in these systems seemed to be too faint for evolutionary expansion to be the dominant driving mechanism. Of course, my physics was lousy and I worked out the mass-loss rates incorrectly. John Faulkner did it right a few years later [17], but his fundamental work was sort of lost when the binary pulsar was discovered and proved to be a "cleaner" demonstration of the existence of gravitational radiation, expecially to those in the physics community.

Speaking of mass-loss driving mechanisms, examples of which are stellar evolution and gravitational-wave grinding, I think it might be of interest to note here the third proposed mechanism, viz., magnetically coupled winds, a process that has been convincingly pressed by Joe Patterson [18]. In this picture, one takes advantage of the expectation that all late-type stars have solar-type winds coupled to magnetic fields, which act to slow the rotational velocity, just as the solar wind slows the rotation of the sun. In a close binary, the tidal couple transfers orbital angular momentum from the orbit to the wind. The stars draw together, and matter flows out through $\mathrm{L}_{1}$. This picture is, of course, a beautiful extension of earlier ideas by E. Schatzman and especially Su-Shu Huang [19] who, in the mid- to late- $60 \mathrm{~s}$, first proposed such a process while some of us were taking the rather minimalist (and essentially wrong) position that dwarf novae (and maybe novae) were descended from W UMa stars. But the joke is on me, I'm afraid, for more-or-less ignoring this possibility. As some of you might recall, I worked a lot on stellar rotation in the ' $60 \mathrm{~s}$ using the coudé spectrographs at Mount Wilson and Palomar. I was able to establish [20] that solar-type stars rotated faster in the Hyades than in old field F's and G's and that Pleiades stars rotated fainter still. Presumably angular momentum was being lost on a $10^{8}-10^{9}$ year timescale, as a result of magnetically-coupled stellar winds. In one of those incredible bursts of blindness that can only be described as perverse, I never saw the connection between the nova binary results and the stellar rotation results. $C^{\prime}$ est la vie, I guess is the only remark I can make!

Let me conclude then with remarks on a few of the things that have impressed me, as I've attempted to re-establish a connection with a field I retired from 20 years ago. This will be selective and presumptuous. And be assured that neglect of something you think is important results from ignorance, not malice. I focus on the binary and evolutionary aspects of the problem, of course, 
and neglect the enormous advances in both observation and theory associated with the origin of the outbursts, (e.g., the superb work of Starffield, Truran and their associates) with expanding nebular shells and their excitation, their abundances, etc. Nor will I attempt to recount the development of a vast storehouse of new information on interacting binaries among cataclysmic variables, largely the work of southern hemisphere astronomers, e.g., B. Warner, N. Vogt, and others. What always interested me most was the evolutionary scenario - where do novae and related objects come from? How do they evolve and die? Are the different kinds of objects - viz., dwarf nova, nova-like, recurrent, classical, - on parallel tracks or does one kind evolve into another? Anyway here's a short list of developments that have amused this old man.

1). X-ray sources and collapsed states of matter more exotic than white dwarfs. What X-ray astronomy showed in the early 70's was that there existed a hitherto unknown class of objects in which material was falling into much deeper potential wells than could be provided by a white dwarf. The X-ray astronomers thus transformed a backwater subject into an exciting frontline activity involving neutron stars and black holes.

2) The recognition - first by Hans Ritter [21], I believe, that you couldn't provide for the rather massive white dwarfs seen in old novae and related objects except through the evolution of binaries of intermediate mass, and that significant losses of angular momentum were required. This spelled the doom of the earlier "minimalist" scenarios.

3). The prediction, through analogy with pulsars, by Bath, Evans and Pringle [22], that the ultra-short period fluctuations of, e.g., DQ Her, resulted from a spinning white dwarf dipole, and the discovery by Kemp and his associates [23] and others of the corresponding magnetic fields. This led to trouble with the accretion disk model, the end of which we have not yet seen, as Bob Williams [24] showed in a recent $A . J$.- no doubt we will hear more about that in this meeting.

4). The recognition that there are "too many" cataclysmics in globular clusters, that the origin of binaries by capture processes must be considered in such an environment, and the possible effect of such processes on the dynamical evolution of clusters.

5). Joe Patterson's masterful 1984 paper, in which the case for magnetic wind and spindown was most cogently made, and in which the challenge of the mismatch between nova and close-binary statistics was pointed out most dramatically.

6). The acceptance of this challenge by Mike Shara and Mario Livio [25] and the proposal of the "hibernation scenario."

I've left out all sorts of important things, no doubt. But I do have a plan for the future and hope to do something worthwhile in this field before I retire. As most of you know, we expect to have the 10-m Caltech/University of California (Keck) Telescope in operation on Mauna Kea by 1992. On the UC side, we have about 150 people who'd like to use it and we expect to get about 150 nights per year as the UC share. I reckon that, if time-resolved spectroscopy at 15 th magnitude was possible on a 10-15 minute timescale using the 200-inch with old-fashioned techniques in 1960, then one should get to 19 th magnitude with the 200 -inch using modern techniques. However, with the $10-\mathrm{m}$ telescope, this could be extended to about $\mathrm{V} \sim 21$. So Richard Stover and I have a plan for our two nights (there are two of us, you see!) with the $10-\mathrm{m}$ in 1992 or 1993 . We will examine Mike Shara's prediction of an orbital period of $P=3^{\text {h }} .6$ in the case of CK Vul! I think it's just possible with the 10-m, but it won't be easy. 


\section{REFERENCES}

[1] Sandage, A. and Schwarzschild, M. 1952, Ap. J., 116, 463.

[2] Crawford, J. 1954, Ap. J., 121, 71; also Crawford, J. and Kraft, R.P. 1956, Ap. J., 123, 44.

[3] Joy, A. H. 1954, Ap. J., 120, 337; also Pub. A.S.P., 66, 5.

[4] Joy, A. H. 1956, Ap. J., 124, 317.

[5] Sanford, R. F. 1948, Ap. J., 109, 81.

[6] Mestel, L. 1952, M.N.R.A.S., 112, 598.

[7] Walker, M. F. 1954, Pub. A.S.P., 66, 71.

[8] Walker, M. F. and Herbig, G. H. 1954, Ap. J., 120, 278.

[9] Walker, M. F. 1954, Pub. A.S.P., 66, 230.

[10] Walker, M. F. 1962, Ap. J., 138, 313.

[11] Kraft, R. P. 1958, Pub. A.S.P., 70, 598.

[12] Greenstein, J. L. and Kraft, R. P. 1959, Ap. J., 130, 99; also Kraft, R.P., Ap. J., 130, 110.

[13] Kraft, R. P. 1958, Ap. J., 127, 625.

[14] Kraft, R. P. 1964, Ap. J., 139, 457.

[15] Kraft, R. P., Mathews, J. and Greenstein, J. L. 1962, Ap. J., 136, 312.

[16] Kraft, R. P. 1966, Trans. I.A.U., XIIB, 519.

[17] Faulkner, J. 1971, Ap. J. (Letters), 170, L99.

[18] Patterson, J. 1984, Ap. J. Suppl., 54, 443.

[19] Huang, Su-Shu 1966, Ann. d'Ap., 29, 331.

[20] Kraft, R. P. 1967, Ap. J., 150, 551.

[21] Ritter, H. 1976, MN.R.A.S., 175, 279.

[22] Bath, G., Evans, W., and Pringle, J. 1974, M.N.R.A.S., 166, 113.

[23] Kemp, J., Swedlund, J. and Wolstencroft, R. 1974, Ap. J. (Letters), 193, L15.

[24] Williams, R. 1989, A. J., 97, 1752.

[25] Livio, M. and Shara, M. 1987, Ap. J., 319, 819; also Shara, M. 1989, Pub. A.S.P., 101, 5. 